\title{
Insulin deficiency induces rat renal mesangial cell dysfunction via activation of IGF-1/IGF-1R pathway
}

\author{
Ya-li KONG ${ }^{1}$, Yang SHEN ${ }^{1}$, Jun NI ${ }^{1}$, De-cui SHAO ${ }^{1}$, Nai-jun $\mathrm{MIAO}^{1}$, Jin-lan XU ${ }^{1}$, Li ZHOU ${ }^{1}$, Hong XUE ${ }^{1}$, Wei ZHANG ${ }^{1}$, Xiao-xia \\ WANG $^{2, *}$, Li-min LU ${ }^{1, *}$
}

${ }^{1}$ Department of Physiology and Pathophysiology, Shanghai Medical College, Fudan University, Shanghai 200032, China; ${ }^{2}$ Department of Nephrology, Shanghai Jiao Tong University, School of Medicine, Shanghai 200025, China

\begin{abstract}
Aim: Diabetic nephropathy is one of the major complications of diabetes and the major cause of end-stage renal disease. In this study we investigated the insulin deficiency (ID) induced changes in renal mesangial cells (MCs) and in the kidney of STZ-induced diabetic rats.

Methods: Cultured rat renal MCs were incubated in ID media. Cell proliferation was analyzed using BrdU incorporation assay. The expression of insulin receptor (IR), insulin-like growth factor-1 receptor (IGF-1R), phosphorylated IGF-1R, fibronectin, and collagen IV was determined with Western blot analysis. STZ-induced diabetic rats were treated with an IGF-1R antagonist picropodophyllin (PPP, $\left.20 \mathrm{mg} \cdot \mathrm{kg}^{-1} \cdot \mathrm{d}^{-1}, \mathrm{po}\right)$ for 8 weeks. After the rats were euthanized, plasma and kidneys were collected. IGF-1 levels in renal cortex were measured with RT-PCR or ELISA. The morphological changes in the kidneys were also examined.

Results: Incubation in ID media significantly increased cell proliferation, the synthesis of fibronectin and collagen IV, and the expression of IGF-1 and IGF-1R and phosphorylated IGF-1R in renal MCs. Pretreatment of the cells with PPP (50 nmol/L) blocked ID-induced increases in cell proliferation and the synthesis of fibronectin and collagen IV; knockdown of IGF-1R showed a similar effect as PPP did. In contrast, treatment of the cells with IGF-1 $(50 \mathrm{ng} / \mathrm{mL})$ exacerbated ID-induced increases in cell proliferation. In the kidneys of diabetic rats, the expression of IGF-1, IGF-1R and phosphorylated IGF-1R were significantly elevated. Treatment of diabetic rats with PPP did not lower the blood glucose levels, but significantly suppressed the expression of TGF- $\beta$, fibronectin and collagen IV in the kidneys, the plasma levels of urinary nitrogen and creatinine, and the urinary protein excretion.

Conclusion: Insulin deficiency increases the expression of IGF-1 and IGF-1R in renal MCs and the kidney of diabetic rats, which contributes to the development of diabetic nephropathy.
\end{abstract}

Keywords: diabetic nephropathy; insulin deficiency; IGF-1R; IGF-1; picropodophyllin; renal mesangial cells; STZ rats

Acta Pharmacologica Sinica (2016) 37: 217-227; doi: 10.1038/aps.2015.128; published online 18 Jan 2016

\section{Introduction}

Diabetes (diabetes mellitus, DM) is a group of metabolic diseases in which blood glucose levels are higher than normal for prolonged periods of time. Diabetic nephropathy (DN) is one of the major complications of DM and is the major cause of end-stage renal disease (ESRD $)^{[1]}$. In the early stage of $\mathrm{DN}$, the kidneys are enlarged. These changes are followed by the overproduction and accumulation of extracellular matrixes (ECM), thickness in the glomerular basal membrane, and final development of glomerular sclerosis and interstitial fibrosis ${ }^{[2]}$. The glomerulus is an important target for DN. Glomerular hyper-

\footnotetext{
${ }^{*}$ To whom correspondence should be addressed.

E-mail lulimin@shmu.edu.cn (Li-min LU); omaha198501@163.com (Xiao-xia WANG)

Received 2015-09-05 Accepted 2015-11-02
}

trophy is one of the main pathological features of early DN. Mesangial cells (MCs) are inherent mesenchymal cells among the glomerular capillary loops. MC proliferation is commonly observed in a variety of pathological changes in glomerular injury. It has been demonstrated that MC dysfunction, including overproliferation and an increase in ECM production ${ }^{[3,4]}$, is implicated in the development of $\mathrm{DN}^{[5,6]}$.

Hyperglycemia has been well documented as an independent risk factor for DM-induced complications. Many previous studies, including the observations in our lab, explored the role of hyperglycemia in $\mathrm{DN}$ and the related mechanisms ${ }^{[7-11]}$. Moreover, many other factors, such as the overactivation of the renin-angiotensin system, oxidative stress, and an elevation in advanced glycation end products, are all reported to be involved in diabetes-induced renal injury.

The fundamental basis of diabetes is functionally insuffi- 
cient insulin, which can be due to either the $\beta$-cells in the pancreas islet not producing enough insulin or an inability of cells in the body to correctly respond to the secreted insulin. In addition to its blood glucose-regulating effect, insulin confers multiple physiological effects by binding to its receptors and interacting with its homologue IGF-1. However, few studies have explored the functional role of a deficiency of insulin in the development and progression of DN. The aim of this study was to identify the role of insulin deficiency (ID) in DN and its underlying mechanism in cultured renal MCs and in STZ-induced DM rats.

\section{Materials and methods Materials and reagents}

Normal-glucose Dulbecco's modified Eagle's medium (DMEM) and STZ were purchased from Sigma-Aldrich (St Louis, MO, USA). Steroid hormone-free fetal bovine serum (FBS) was purchased from Sijiqing Biological Engineering Materials Co (Hangzhou, China). Insulin was from Eli-Lilly (Indianapolis City, IN, USA). The recombinant murine IGF-1 was from Peprotech (Rocky Hill, NJ, USA). Picropodophyllin (PPP) was from Millipore (Billerica, MA, USA) and MedChemExpress (Monmouth Junction, NJ, USA). The BCA Protein Assay Kit was from Shenergy Biocolor BioScience and Technology (Shanghai, China). The RNA extraction kit was from Sangon Co (Shanghai, China). The ReverTra AceqPCR RT kit and the SYBR Green reaction mix were from Toyobo Co (Dojima Hama, Osaka, Japan). The BrdU assay kit was purchased from Roche (Mannheim, Germany). The enhanced chemiluminescence (ECL) detection kit was from Pierce Biotechnology (Rockford, IL, USA). The rabbit anti-IR antibody and the rabbit anti-IGF-1R antibody were obtained from Santa Cruz Biotechnology, Inc (Dallas, TX, USA). The phosphoIGF-1 receptor (Tyr1135/1136) was obtained from Cell Signaling Technology (Beverly, MA, USA). The anti-fibronectin antibody was obtained from Sigma-Aldrich (St Louis, MO, USA), the anti-collagen IV antibody was from Abcam (Cambridge, MA, USA), the rabbit anti-rat TGF- $\beta$ (Y369) antibody was obtained from Bioworld Technology, Inc (Nanjing, China), and the alpha-tubulin monoclonal antibody was from Proteintech (Chicago, IL, USA). All of the other chemicals and reagents used in the experiment were of analytical grade.

\section{Cell culture}

The rat renal mesangial cell line (HBZY-1) was purchased from the China Center for Type Culture Collection (Wuhan, China) and cultured in normal DMEM media containing 10\% FBS and $100 \mu \mathrm{U}$ insulin under an atmosphere of $5 \% \mathrm{CO}_{2}$ at $37^{\circ} \mathrm{C}$. Before the experiment, the cells were starved in a medium containing $0.5 \%$ FBS and $100 \mu \mathrm{U}$ insulin for $12 \mathrm{~h}$ to obtain quiescent cells. The ID media was made by supplementing the normal DMEM media without insulin. When necessary, the cells were pretreated with a diluent (DMSO) or PPP at different concentrations for $60 \mathrm{~min}$ before ID application. Each experiment was repeated at least three times.

\section{Animal model}

Age-matched 4-month-old male SD rats weighing 180-210 g were provided by the Shanghai SLAC Laboratory Animal Center. All of the experimental procedures followed the Criteria of the Medical Laboratory Animal Administrative Committee of Shanghai and the Guide for Care and Use of Laboratory Animals of Fudan University, and were approved by the Ethics Committee for Experimental Research, Shanghai Medical College, Fudan University. The animals were acclimatized for 7 days before the study and had free access to water and standard rat chow throughout the experiment. Diabetes was induced by a single intraperitoneal injection of STZ (60 $\mathrm{mg} / \mathrm{kg}$ ) dissolved in $0.1 \mathrm{~mol} / \mathrm{L}$ sodium citrate buffer $(\mathrm{pH} 4.0)$. Seventy-two hours later, the diabetic state was confirmed by measuring blood glucose from the rat tail, using a blood glucose meter (Abbott, Chicago, IL, USA) and One Touch strips (range $=0-27.8 \mathrm{mmol} / \mathrm{L}$ ). Only those rats with plasma glucose concentrations $>16.7 \mathrm{mmol} / \mathrm{L}$ were recruited in the study. After 4 weeks of cultivation, the rats were randomly divided into four groups ( $n=10$ for each group): (1) control rats (Con), injected with vehicle $(0.1 \mathrm{~mol} / \mathrm{L}$ sodium citrate buffer, $\mathrm{pH} 4.0)$; (2) diabetic rats (DM); (3) diabetic rats with IGF-1R blockade PPP (DM+PPP) $\left(20 \mathrm{mg} \cdot \mathrm{kg}^{-1} \cdot \mathrm{d}^{-1}, p o\right)$; and (4) diabetic rats with insulin (DM+insulin) (10 U, twice/day, hypodermic injection). The drugs were administered to the rats for 8 weeks. Twelve weeks after the STZ injection, the rats were killed, and the plasma and kidneys were removed and kept at $-80^{\circ} \mathrm{C}$ until used.

\section{Cell proliferation assay}

A total of $1 \times 10^{3}$ cells per well were cultured in 96-well plates. When the cells reached $50 \%$ confluence, they were serum starved for $12 \mathrm{~h}$ and then treated with the ID media, IGF-1 or PPP. After $48 \mathrm{~h}$, cell proliferation was assessed by BrdU incorporation assay as previously described ${ }^{[12]}$. Briefly, $10 \mu \mathrm{mol} / \mathrm{L} \mathrm{BrdU}$ labeling solution was added to the medium, which was incubated at $37^{\circ} \mathrm{C}$ for $4 \mathrm{~h}$. Then, the cells were fixed, denatured and incubated in an anti-BrdU-POD (peroxidase) antibody for $90 \mathrm{~min}$ at room temperature. At the end of incubation, the cells were rinsed with phosphate-buffered solution (PBS) at pH 7.0 three times to remove any excess antibody, and then $100 \mu \mathrm{L}$ of the substrate solution was added to each well. After a $30 \mathrm{~min}$ incubation at room temperature, the absorbance of the samples was measured on a TECAN Infinite M200 microplate reader (Salzburg Umgebung, Salzburg, Austria) at $370 \mathrm{~nm}$, while the absorbance obtained at $492 \mathrm{~nm}$ served as a reference value.

\section{Western blotting}

Renal cortex or cultured renal MCs were lysed in $1 \times$ sodium dodecyl sulfate (SDS) supplemented with proteinase inhibitor at a dilution of 1:25. Protein concentrations were determined with the BCA Protein Assay Kit according to the manufacturer's instruction. Approximately $50 \mu \mathrm{g}$ of protein was loaded in each well and separated on a $10 \%$ sodium dodecyl sulfate- 
polyacrylamide gel and then electrophoretically transferred to polyvinylidene difluoride membranes at $270 \mathrm{~mA}$ for $120 \mathrm{~min}$. The membranes were blocked with $5 \%$ skim milk in Tris-buffered saline (TBS) and $0.1 \%$ Tween (TBS/Tween) for one hour at room temperature with gentle rocking. The membranes were incubated in primary antibody overnight at $4^{\circ} \mathrm{C}$ (anti-collagen IV antibody, 1:1000; anti-fibronectin antibody, 1:10000; anti-IR antibody, 1:1000; anti-IGF-1R antibody, 1:1000; antirat TGF- $\beta$ antibody, 1:1000; anti-phospho-IGF-1 receptor antibody, 1:1000; anti-tubulin antibody, 1:10000). The membranes were incubated with a secondary anti-rabbit/mouse antibody (1:1000) for $2 \mathrm{~h}$ at room temperature after three washes with TBS/Tween. The hybridization signals were developed using the ECL detection kit according to the manufacturer's instructions and then detected and quantified using the ImageQuant LAS4000 mini system (GE Healthcare, UK). Alpha-tubulin was used as an internal standard to normalize the protein amounts. The relative intensity of the bands exposed on the films was quantified using Smart Viewer software (Furi Technology Co, Shanghai, China). The average relative protein level in the control group was defined as 1.0.

\section{RNA extraction, cDNA synthesis and quantitative real-time PCR}

Total RNA from renal MCs was isolated with TRIzol reagent, and the RNA concentration was measured at the specific absorbance at $260 \mathrm{~nm}$. Two micrograms of total RNA was used for cDNA synthesis in a $20-\mu \mathrm{L}$ reaction mixture with the ReverTra Ace qPCR RT Kit. SYBR Green qRT-PCR was used to quantify the relative abundance of IGF-1 mRNA. The PCR primers are: forward primer 5'-TAGGTGGTTGATGAATGGT-3', reverse primer 5'-GAAAGGGCAGGGCTAAT $-3^{\prime}$ for IGF-1; and forward primer $5^{\prime}$ - CCCTTCATTGACCTCAACTACATG-3', reverse primer 5' - CTTCTCCATGGTGGTGAAGAC-3' for GAPDH, which was used as an endogenous control to normalize the amount of RNA. The PCR procedures were as follows: pre-denaturing at $95{ }^{\circ} \mathrm{C}$ for 5 min followed by 40 cycles of amplifications by denaturing at $95^{\circ} \mathrm{C}$ for $15 \mathrm{~s}$; annealing for $30 \mathrm{~s}$ at $64^{\circ} \mathrm{C}$ (for IGF-1) or $60^{\circ} \mathrm{C}$ (for GAPDH); and extension at $72{ }^{\circ} \mathrm{C}$ for $30 \mathrm{~s}$. After a final extension at $72{ }^{\circ} \mathrm{C}$ for $10 \mathrm{~min}$, the amplified products were subjected to a stepwise increase in temperature from $55{ }^{\circ} \mathrm{C}$ to $95^{\circ} \mathrm{C}$ to construct dissociation curves. The average relative mRNA in the control group was defined as 1.0.

\section{SiRNA transfection}

IGF-1R siRNA was synthesized by Biotend Company (Shanghai, China), with a sequence of 5'-GCCGACACUACUACUACAATT-3'. A nonsilencing siRNA oligonucleotide that does not recognize any known homolog of mammalian genes (Biotend, Shanghai, China) was used as a negative control. MCs were transfected with IGF-1R siRNA $(50 \mathrm{nmol} / \mathrm{L})$ or control siRNA ( $50 \mathrm{nmol} / \mathrm{L}$ ) facilitated by Lipofectamine 2000 transfection reagent (Invitrogen, Carlsbad, CA, USA) according to the manufacturer's instructions. In brief, before transfection, the Lipofectamine 2000 reagent was incubated in OPTI-MEM medium (Gibco, Carlsbad, CA, USA) for $5 \mathrm{~min}$ at room tem- perature. At the same time, siRNA was diluted into OPTIMEM medium for $5 \mathrm{~min}$ at room temperature. Next, the diluted Lipofectamine 2000 transfection reagent and diluted siRNA were gently mixed and incubated for $20 \mathrm{~min}$ at room temperature to form siRNA/Lipofectamine 2000 Transfection Agent transfection complexes. IGF-1R protein and cell proliferation were analyzed $48 \mathrm{~h}$ after transfection.

\section{Plasma creatinine, blood urea nitrogen (BUN), urinary protein and glucosuria}

Glucosuria was detected using the oxidase method by employing a glucosuria detection kit (Applygen, Beijing, China). Plasma creatinine, BUN and urinary protein were detected with a creatinine detection kit, BUN detection kit and urinary protein detection kit, respectively (Nanjing Jiancheng Bioengineering Institute, Nanjing, China). All of the kits were used in accordance with the manufacturer's instructions.

\section{Quantification of IGF-1 in renal cortex homogenates by ELISA}

At the end of the experiment (12 weeks of diabetes), the rats were euthanized, and the renal cortex was harvested and stored at $-80^{\circ} \mathrm{C}$ until they were used for the IGF-1 assay by ELISA, which was performed according to the manufacturer's instructions (R\&D Systems, Minneapolis, MN, USA). Briefly, the renal cortex homogenates were diluted 5-fold and then transferred to microplates coated with a monoclonal antiIGF-1. After a $2 \mathrm{~h}$ incubation at room temperature on a horizontal orbital microplate shaker, the wells were aspirated and washed 5 times with wash buffer. This was followed by incubation with the HRP conjugated anti-IGF-1 antibody for another $2 \mathrm{~h}$, and then, the substrate for HRP was added to each well, with subsequent incubation for $30 \mathrm{~min}$ at room temperature on the benchtop, protected from light. Finally, the chromogenic reaction was halted with a stop solution, the plate was gently tapped to ensure thorough mixing, and the absorbance at $540 \mathrm{~nm}$ was measured using a TECAN Infinite M200 microplate reader.

\section{Renal histology and electron microscopy}

At the end of the experiment, the kidneys were collected and fixed in 10\% neutral buffered formalin and then embedded in paraffin. The paraffin sections $(3 \mu \mathrm{m})$ were prepared and stained with HE, PAS and Masson's trichrome. Histological changes, such as the degree of glomerular injury and renal fibrosis, were observed under $400 \times$ optical magnification. Part of the right kidney was fixed in $2.5 \%$ glutaraldehyde for electron microscopy. The ultrastructure (glomerulus basement membrane and podocyte processes) of the kidney was observed at magnifications of $\times 1500$ and $\times 4000$, respectively.

\section{Statistical analysis}

The data are expressed as the mean \pm SEM. The statistical analyses were performed using SPSS Statistics 17.0 software (SPSS, Inc, Chicago, IL, USA). The results of three or more groups were analyzed by one-way ANOVA. The differences between two groups were compared by independent samples 
t-test. All of the group data were tested for normal distributions before statistical comparisons. $P<0.05$ was considered a statistically significant difference between groups.

\section{Results}

Effects of ID on mesangial cell proliferation and ECM synthesis

MCs were cultured in a medium containing different concen-

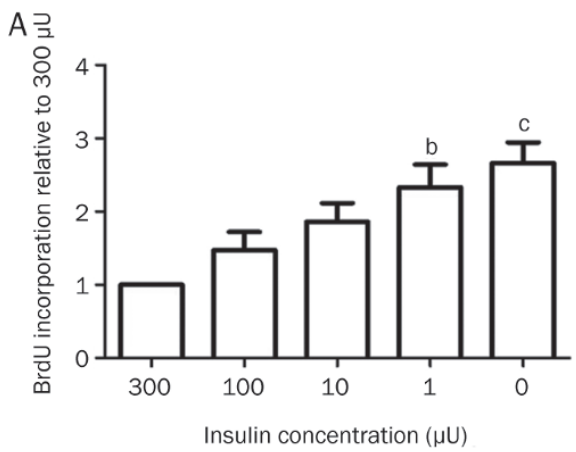

B
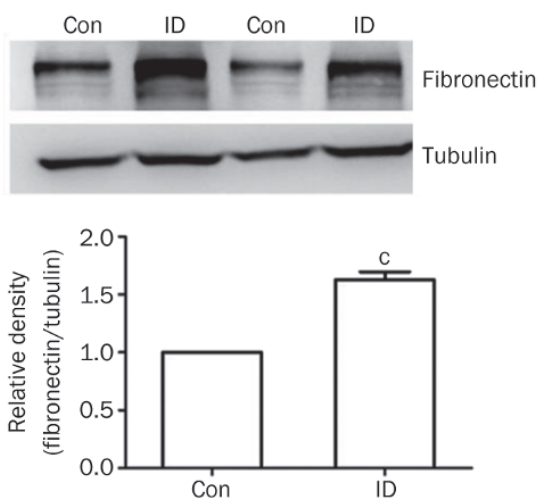

C
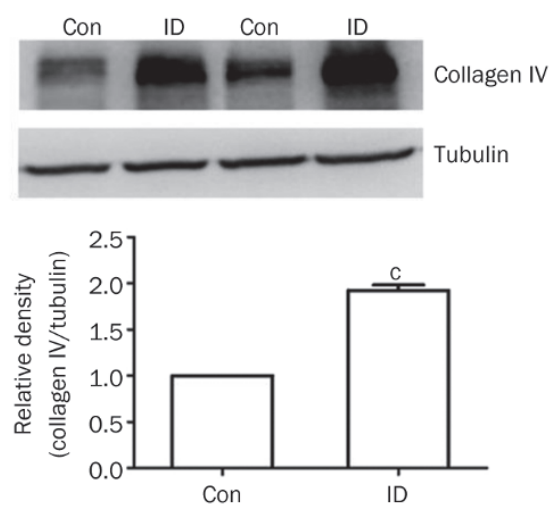

Figure 1. Effects of insulin deficiency (ID) on cell proliferation and ECM synthesis in cultured renal MCs. (A)Cell proliferation was measured by BrdU incorporation assay. The cells were cultured in different concentrations of insulin $(300-0 \mu \mathrm{U})$ for $48 \mathrm{~h}$. The data are expressed as the mean \pm SEM of 8 experiments. ${ }^{b} P<0.05,{ }^{\circ} P<0.01$ vs insulin 300 $\mu \mathrm{U}$. The relative protein levels of fibronectin (B) and collagen IV (C) were analyzed by Western blot. The data are expressed as the mean \pm SEM of 3 experiments. ${ }^{c} P<0.01$ vs the control [insulin $(100 \mu \mathrm{U})$ ]. trations of insulin $(300-0 \mu \mathrm{U})$. A BrdU incorporation assay was used to evaluate cell proliferation. A decrease in insulin concentration induced an increase in cell proliferation (Figure 1A). After depriving the cells of insulin for $48 \mathrm{~h}$, the Western blot results showed that ECM protein levels, including fibronectin (Figure 1B) and collagen IV (Figure 1C), were significantly increased.

Effects of ID on insulin receptor (IR), IGF-1R and IGF-1 expression in renal MCs

After insulin deprivation for $48 \mathrm{~h}$, the Western blot results

A
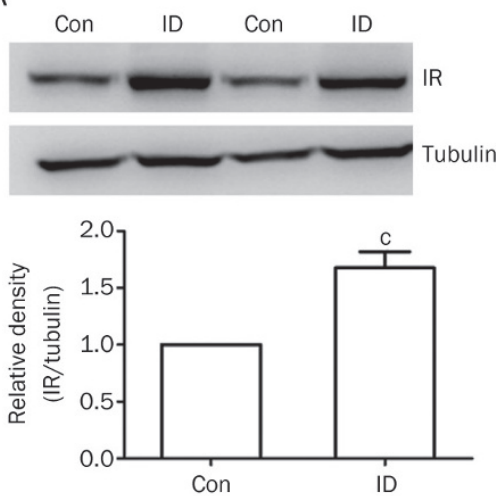

B

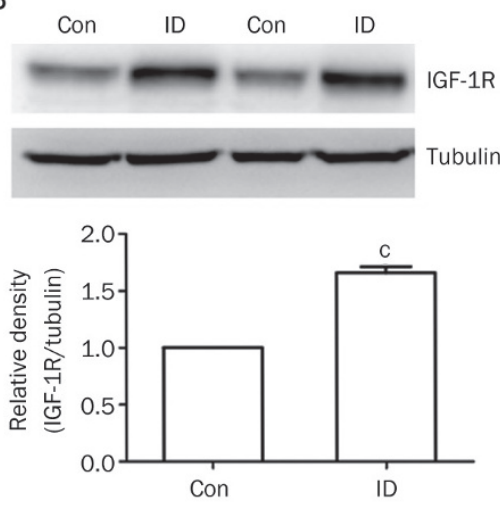

C

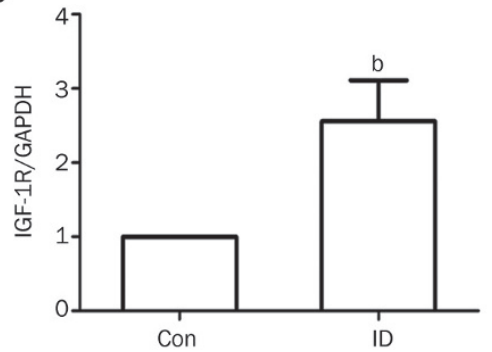

Figure 2. Effects of insulin deficiency (ID) on insulin receptor (IR), IGF-1R and IGF-1 expression in renal MCs. The cells were deprived of insulin for $48 \mathrm{~h}$. The relative protein levels of IR (A) and IGF-1R (B) were analyzed by a Western blot assay. (C) The IGF-1 mRNA levels were analyzed by realtime PCR. The data are expressed as the mean \pm SEM of 3 experiments. ${ }^{\mathrm{b}} P<0.05,{ }^{\mathrm{c}} P<0.01$ vs the control [insulin $(100 \mu \mathrm{U})$ ]. 
showed that the expression of both IR (Figure 2A) and IGF$1 \mathrm{R}$ (Figure 2B) was significantly increased in MCs; the RT-PCR results showed that the IGF-1 mRNA level was also significantly increased (Figure 2C).

The IGF-1R antagonist PPP inhibited ID-induced cell proliferation and increase in ECM synthesis in renal MCs

To examine whether the change in the IGF-1 and IGF-1R pathway was involved in ID-induced MCs proliferation and ECM synthesis, PPP (an IGF-1R antagonist) was utilized in the experiment. The Western blot showed that the phosphorylation of IGF-1R was significantly increased in the insulindeprived MCs. PPP reversed the ID-induced up-regulation in IGF-1R phosphorylation (Figure 3A and Figure 3B). The BrdU incorporation assay showed that the blockade IGF-1R with PPP reduced ID-induced MC proliferation (Figure 3C). Similarly, the increase in fibronectin (Figure 3D) and collagen IV (Figure 3E) synthesis in the ID MCs was also inhibited by PPP treatment.
Effects of IGF-1R knock-down on ID-induced cell proliferation in renal MCs

IGF-1R siRNA (50 nmol/L) transfection (48 h) significantly knocked down the expression of IGF-1R, while the transfection of the negative control (NC) siRNA (50 nmol/L) did not have an obvious effect (Figure 4A). ID elevated MC proliferation. IGF-1R siRNA (50 nmol/L) transfection significantly inhibited the elevation of MC proliferation induced by ID (Figure 4B).

\section{Exogenous IGF-1 increased MC proliferation}

The BrdU incorporation assay showed that compared with the control group, the ID group induced an increase in MC proliferation, and cells treated with IGF-1 (50 ng/mL) showed increased MC proliferation. In the insulin-deprived cells, the application of IGF-1 induced an even higher increase in cell proliferation (Figure 4C).

\section{Effects of IGF-1R blockade on physiological parameters in} diabetic rats

As illustrated in Figure 5, the body weights of the DM,
A
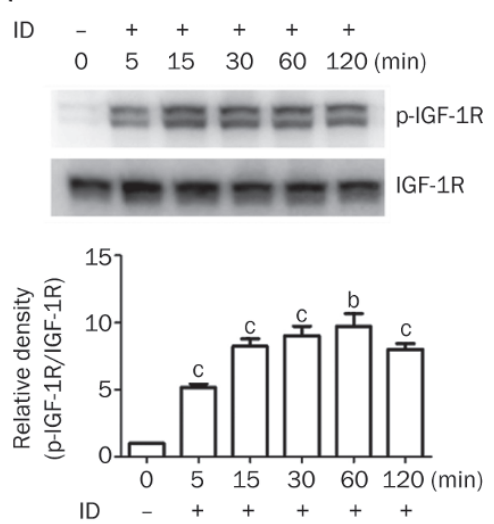

D
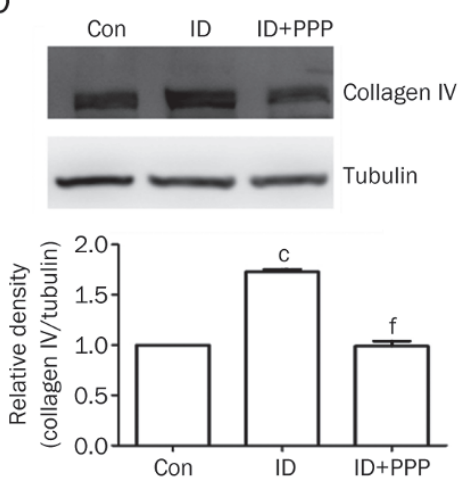

B $\begin{array}{lllllll}\mathrm{ID} & - & + & + & + & + & + \\ \operatorname{PPP}(\mathrm{nmol} / \mathrm{L}) & 0 & 0 & 50 & 100 & 200 & 500\end{array}$
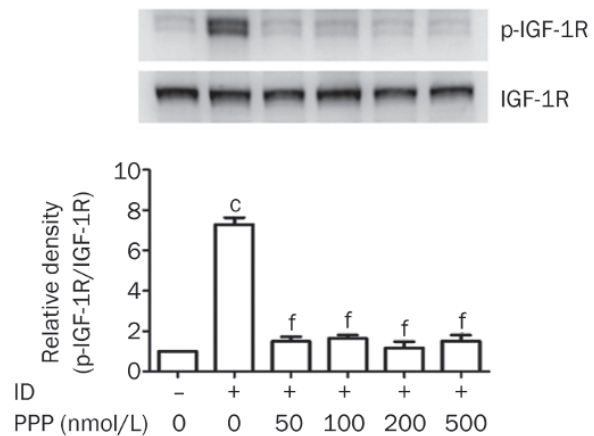

E
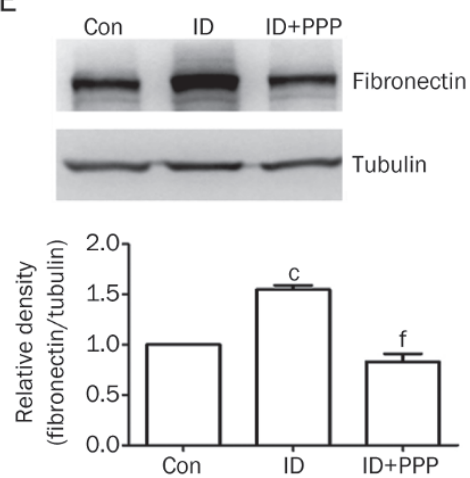

Figure 3. IGF-1R antagonist PPP inhibited insulin deficiency (ID)-induced cell proliferation and increase in ECM synthesis in renal MCs. (A) The cells were treated with ID for 0-120 min. Phospho-IGF-1R (Tyr1135/1136) was analyzed by a Western blot assay. The data are expressed as the mean \pm SEM of 4 experiments. ${ }^{b} P<0.05,{ }^{c} P<0.01$ vs the control [ID $(0$ min)]. (B) The phosphorylation of IGF-1R was observed by a Western blot assay after the cells were pretreated with PPP $50 \mathrm{nmol} / \mathrm{L}$ or a diluent for $60 \mathrm{~min}$ and deprived of insulin for $5 \mathrm{~min}$. (C) Cell proliferation was measured by a BrdU incorporation assay after the cells were pretreated with PPP $50 \mathrm{nmol} / \mathrm{L}$ or a diluent for $60 \mathrm{~min}$ and deprived of insulin for $48 \mathrm{~h}$. The relative protein levels of fibronectin (D) and collagen IV (E) were analyzed by Western blot assay. The data are expressed as the mean \pm SEM of 8 experiments (C) and 4 experiments (D and $E) .{ }^{c} P<0.01$ vs the control $\left[I D(-)\right.$ and PPP (-)]. ${ }^{f} P<0.01$ vs ID only. 
A
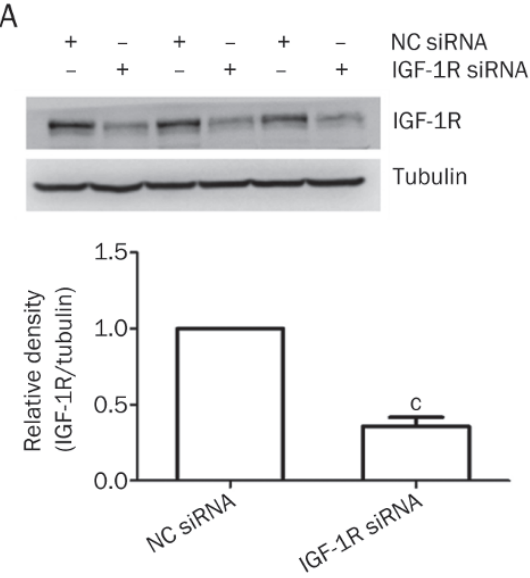

B

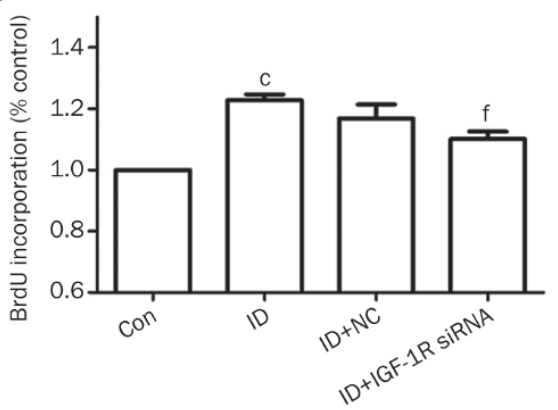

C

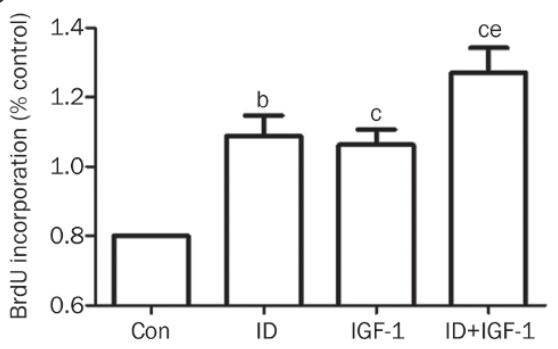

Figure 4. Effect of IGF-1R siRNA transfection on insulin deficiency (ID)induced cell proliferation and the effect of exogenous IGF-1 on the proliferation of renal MCs. (A) The protein level of IGF-1R was detected by a Western blot assay after the MCs were transfected with an IGF-1R siRNA $(50 \mathrm{nmol} / \mathrm{L})$ or a control siRNA $(50 \mathrm{nmol} / \mathrm{L})$ for $48 \mathrm{~h}$. (B) The effect of the IGF-1R siRNA on ID-induced proliferation in renal MCs. Cell proliferation was measured by a BrdU incorporation assay after siRNA transfection for $48 \mathrm{~h}$. (C) The effect of exogenous IGF-1 on the proliferation of renal MCs. Cell proliferation was measured by a BrdU incorporation assay after the MCs were treated with ID or IGF-1 $(50 \mathrm{nmol} / \mathrm{L})$ for $48 \mathrm{~h}$. The data are expressed as the mean \pm SEM of 3 experiments $(A)$ and 8 experiments ( $B$ and $C$ ). ${ }^{b} P<0.05,{ }^{c} P<0.01$ vs the control. ${ }^{e} P<0.05,{ }^{f} P<0.01$ vs ID [ID and NC siRNA)].

$\mathrm{DM}+\mathrm{PPP}$ and $\mathrm{DM}+$ insulin groups were significantly lower than in the control animals (Figure 5A), while the urine volumes were significantly increased (Figure 5B). The ratio of kidney weight to body weight was significantly higher in the DM animals than in the control rats, while the DM rats treated with PPP or insulin showed a decreased ratio of kidney weight to body weight but it was still significantly higher than in the control rats (Figure 5C). Blood glucose was significantly elevated in the DM rats (Figure 5D). A decrease in blood glucose was observed in the insulin-supplemented DM rats at $4 \mathrm{~h}$ after insulin injection but not at $12 \mathrm{~h}$ (Figure 5E). Urinary glucose was not detected in the control rats. DM rats treated with PPP or insulin tended to show a decrease in urinary glucose output, but obvious urinary glucose was still detected in both the $\mathrm{DM}+\mathrm{PPP}$ and DM+insulin groups (Figure 5F).

Changes in the IGF-1 and IGF-1R protein and phosphorylation levels in the kidney tissues of diabetic rats

Western blot analysis showed that IGF-1R protein (Figure 6A) and phosphorylated IGF-1R (Figure 6B) were significantly increased in DM rats when compared to normal rats. Either administration of the IGF-1R blocker PPP or insulin supplementation significantly reduced IGF-1R expression and its phosphorylation. The ELISA results also showed that the IGF-1 level (Figure 6C) was significantly increased in the DM rats. Treatment of the diabetic rats with either PPP or insulin significantly reduced the IGF-1 level in the DM rats.

\section{IGF-1R blockade on renal function in diabetic rats}

The $24 \mathrm{~h}$ urinary protein (Figure 7A), plasma BUN (Figure 7B) and plasma creatinine (Figure $7 \mathrm{C}$ ) were significantly increased in the DM group at the end of the experiment (12 weeks after the STZ injection). Significant reductions in the $24 \mathrm{~h}$ urinary protein, plasma BUN and plasma creatinine were observed in the DM rats treated with the IGF-1R blockade PPP or insulin.

Blocking IGF-1R alleviated the renal fibrotic response and reduced TGF- $\beta$ protein levels in the kidney

The Western blot results showed that the expression of fibronectin (Figure 8A), collagen IV (Figure 8B) and TGF- $\beta$ (Figure $8 \mathrm{C}$ ) were significantly increased in the DM rats compared to the normal group. Significant reductions in the expression of fibronectin, collagen IV, and TGF- $\beta$ were observed in the DM rats treated with the IGF-1R blockade PPP or insulin.

\section{Effects of IGF-1R blockade on renal morphology in normal and diabetic rats}

HE staining (Figure 9A) showed that there was mild expansion in the glomerular mesangial area and mesangial cell proliferation, and basement membrane thickening in the DM rats. The PAS stain showed that there were sporadic KimmelstielWilson nodules, and few instances glomerular basement membrane thickening, mesangial matrix accumulation and plasma protein extravasation in the DM group (Figure 9B). The Masson staining (Figure 9C) showed that there was a slight increase in ECM deposition in the DM rats. The electron microscopy (Figure 9D) results showed that there was local thickness in the glomerular basement membrane and partial epithelial foot processes fusion in the DM rats. The pathological morphology showed some improvement in the DM+PPP and the DM+insulin groups. 
A

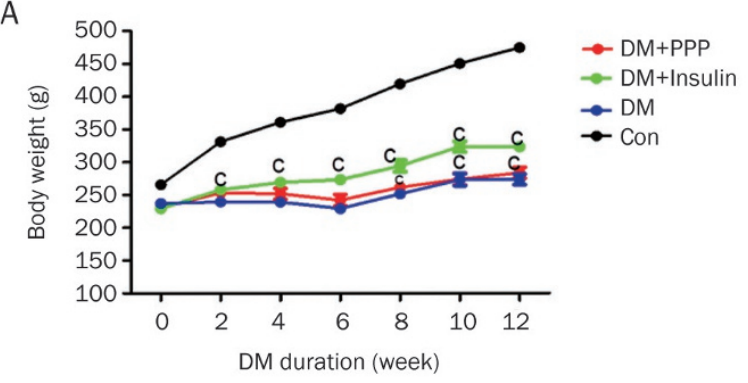

C

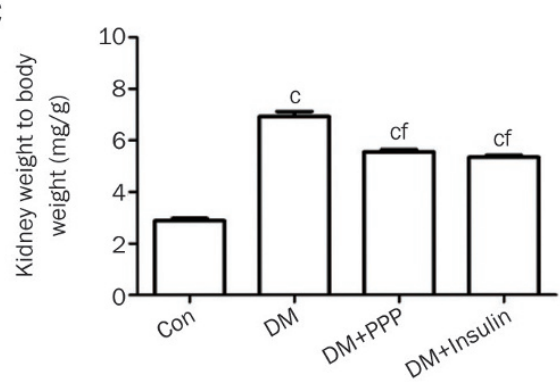

E

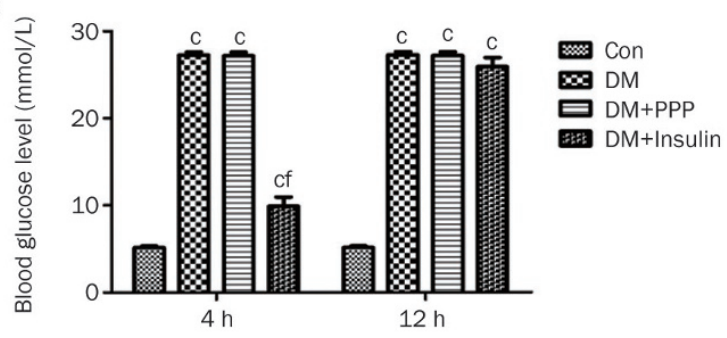

B

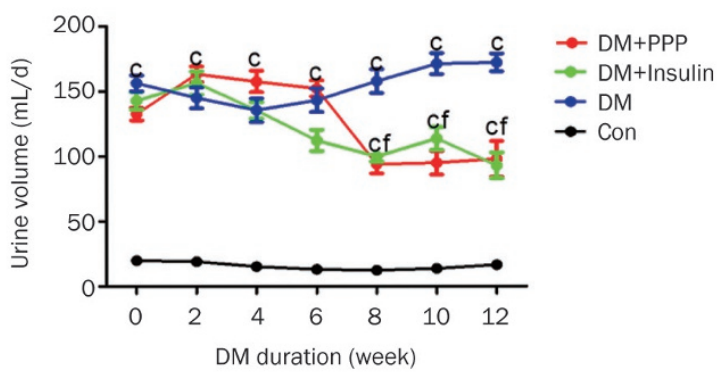

D

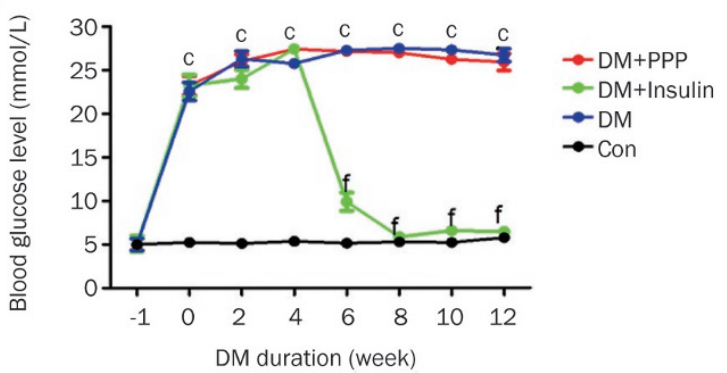

F

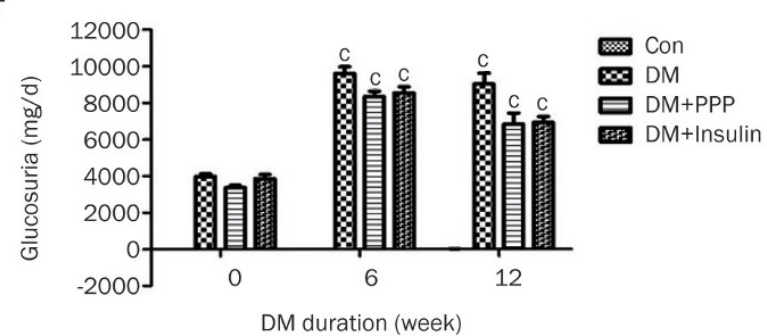

Figure 5. Effects of IGF-1R blockade on the physiological parameters in the diabetic rats. (A) Body weight and (B) urine volume were measured every two weeks after the STZ injection. (C) Twelve weeks after the STZ injection, the rats were euthanized, the kidneys were collected, weighed and the ratio of kidney weight to body weight was calculated. (D) Blood glucose was measured one week before the STZ injection and every two weeks after the STZ injection. (E) Six weeks after the STZ injection, the blood glucose level was measured after insulin administration for $4 \mathrm{~h}$ and $12 \mathrm{~h}$. (F) Glucosuria was detected by a glucosuria detection kit before the STZ injection and 6 and 12 weeks after the STZ injection. The data are expressed as the mean \pm SEM of 7 experiments (B and F) and 10 (A, C, D and E) experiments. ${ }^{\circ} P<0.01$ vs Con. ${ }^{f} P<0.01$ vs DM.

\section{Discussion}

$\mathrm{DM}$ is a common endocrine metabolic disorder syndrome. With the changes in people's lifestyles, the prevalence of DM is continuously increasing. DM has become a growing public health problem of global concern ${ }^{[13]}$. As one of the most common complications of diabetes, DN is a leading cause of endstage renal disease. The initial phase of $\mathrm{DN}$ is characterized by enlargement in the kidneys. At the early stage of $\mathrm{DN}$, basic pathological changes in the glomeruli include thickening of the basement membrane, ECM accumulation and microcirculation disorder. All of the changes will lead to glomerular sclerosis and ultimately contribute to renal functional regres$\operatorname{sion}^{[2]}$. MCs are inherent mesenchymal cells among the glomerular capillary loops. The activation and proliferation of the MCs is involved in a variety of factor-induced pathological changes in glomerular injury. Abnormalities in the MCs cause increases in ECM expression and cytokine secretion, which play an important role in glomerular sclerosis ${ }^{[14]}$. Therefore, MCs were utilized as a target cell in the in vitro studies.

Over the past few decades, numerous studies have explored the pathophysiological mechanism of DN. Multi-factors, such as hyperglycemia ${ }^{[10,15]}$, excessive activation of the RAS system $^{[9]}$, increased oxidative stress ${ }^{[16]}$ and accumulation of advanced glycation end-products (AGEs) ${ }^{[17,18]}$ are all reported to contribute to the development of DN.

Radically, diabetes is a far-reaching pathophysiologic effect of $\mathrm{ID}^{[19,21-24]}$, which may be caused by $\beta$-cells in the pancreas islet that do not produce enough insulin or cells of the body that do not correctly respond to secreted insulin ${ }^{[2,19,20]}$. In addition to the hypoglycemic effect, insulin has multiple effects on metabolism and cell growth. Currently, few studies have investigated the role of ID in the development and progression of DN. The normal physiological insulin concentration is approximately $5-15 \mu \mathrm{U} / \mathrm{mL}$, and it increases five- 
A
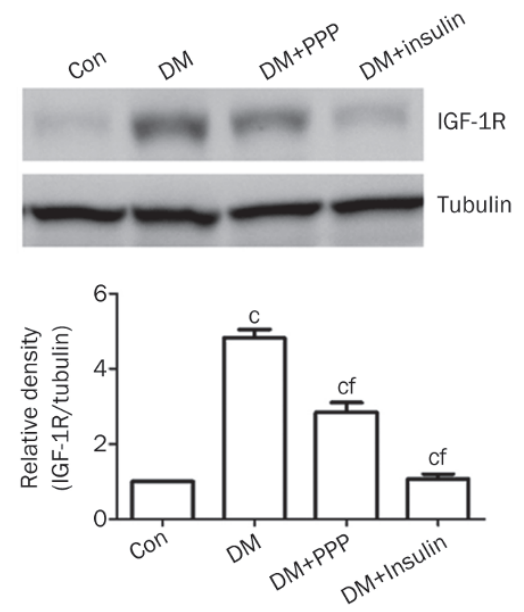

B
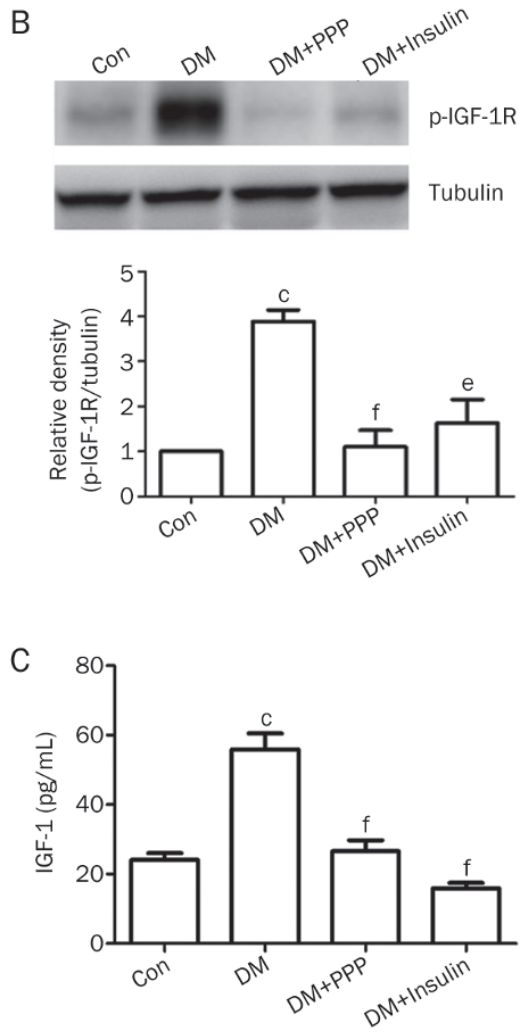

Figure 6. Changes in IGF-1 and IGF-1R protein and IGF-1R phosphorylation levels in diabetic rat kidney tissues. At the end of the study (12 weeks after the STZ injection), the protein levels of IGF-1R (A) and p-IGF-1R (B) were analyzed by a Western blot assay. (C) IGF-1 protein levels were analyzed by ELISA. The data are expressed as the mean \pm SEM of 6 experiments. ${ }^{\mathrm{c}} P<0.01$ vs Con. ${ }^{\mathrm{e}} P<0.05,{ }^{\mathrm{f}} P<0.01$ vs $\mathrm{DM}$.

to ten-fold after eating a meal. To determine the direct role of ID, we cultured MCs in an insulin-deprived condition. In the study, we observed that insulin deprivation induced increased MC proliferation and collagen IV and fibronectin synthesis, which are the major components of the ECM. These results suggested that ID can directly lead to the overproliferation of mesangial cells and ECM overproduction and is one of the
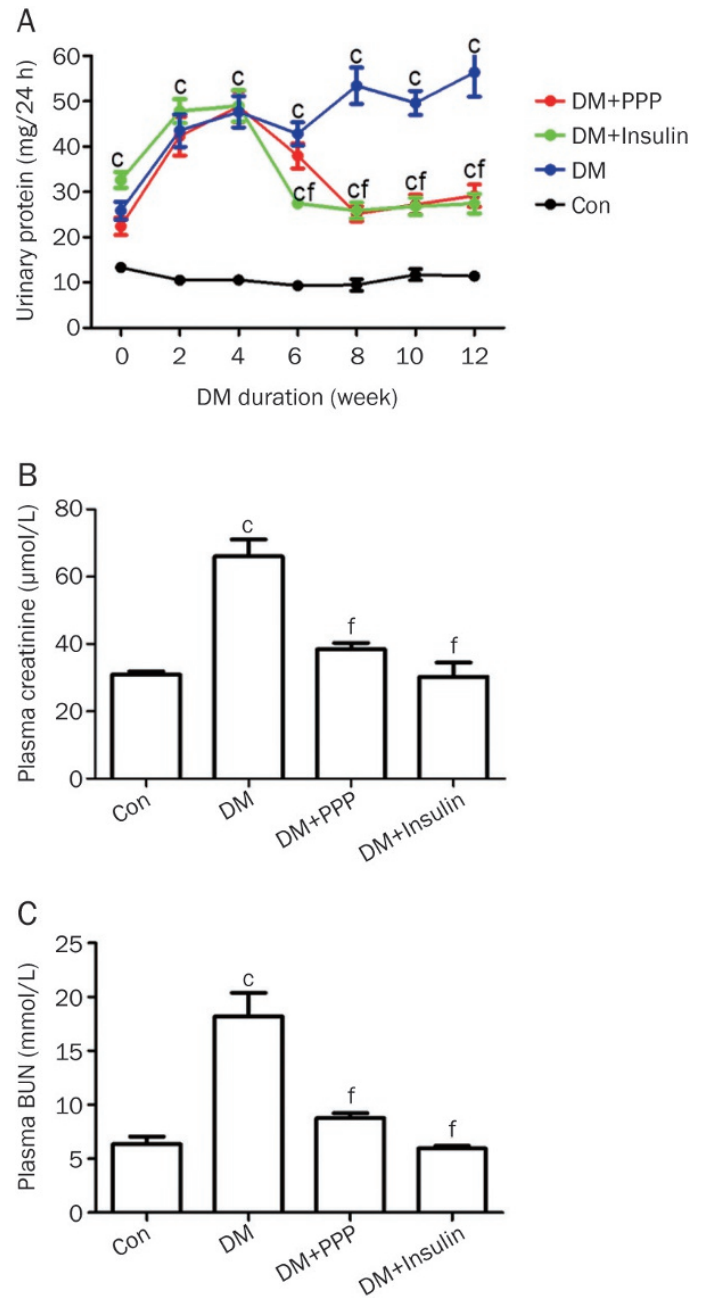

Figure 7. Effects of IGF-1R blockade on renal function in diabetic rats. (A) The urinary protein was detected by a urinary protein detection kit every two weeks after the STZ injection. At the end of the study (12 weeks after STZ injection), plasma creatinine (B), and plasma BUN (C) were detected, respectively. The data are expressed as the mean \pm SEM of 6 experiments (A) and 10 experiments (B and $C$ ). ${ }^{c} P<0.01$ vs Con. ${ }^{f} P<0.01$ vs DM.

direct reasons for cellular functional changes.

IGF-1 is the homolog of insulin, and it plays an important role in regulating the cell cycle and growth ${ }^{[25]}$. IGF-1R also has a structure similar to IR. They all belong to the kinase receptor family ${ }^{[26]}$. IGF-1R activation affects the cell cycle and promotes cell division, differentiation and proliferation. In recent years, many studies have confirmed that the alteration and functional changes of the IGF-1R pathways, by activating the MAPK and PI3K pathways, are closely related to tumor development. IGF-1R overexpression is observed in breast cancer, colon cancer, lung cancer, prostate cancer and pancreatic tumors. As a result, inhibition of the IGF-1R pathway has become one of therapeutic targets for the treatment of cancer, and there are studies showing that inhibition of IGF-1R can effectively alleviate the occurrence and development of tumors ${ }^{[26-33]}$. Abnormal proliferation in MCs and ECM accumulation are the main pathological changes of DN. Previous studies have found 
A
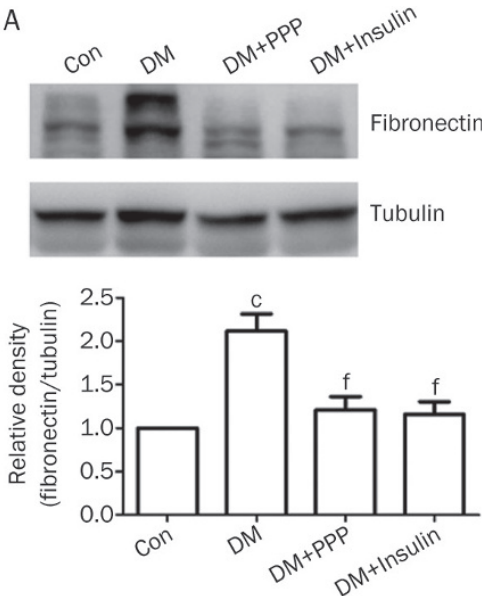

B
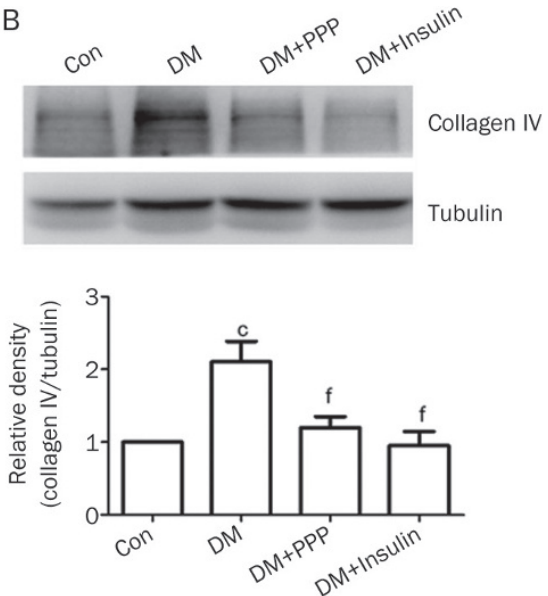

C
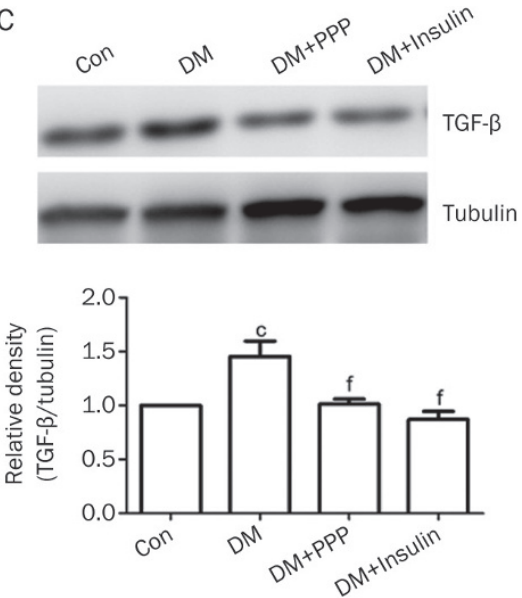

Figure 8. Effects of IGF-1R blockade on ECM synthesis and TGF- $\beta$ protein levels in diabetic rats. At the end of the study (12 weeks after the STZ injection), a Western blot revealed the protein levels of fibronectin (A), collagen IV (B) and TGF- $\beta$ (C). The data are expressed as the mean \pm SEM of 6 experiments. ${ }^{c} P<0.01$ vs Con. ${ }^{f} P<0.01$ vs DM.

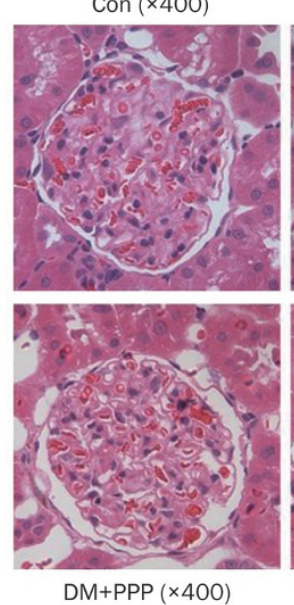

C

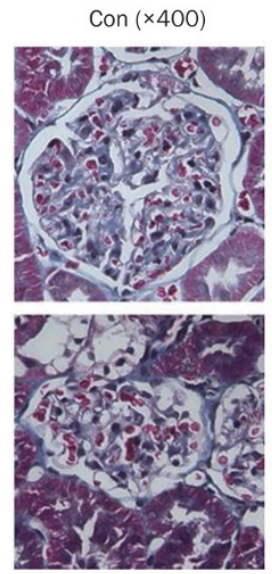

$\mathrm{DM}+\mathrm{PPP}(\times 400)$
$\mathrm{DM}(\times 400)$

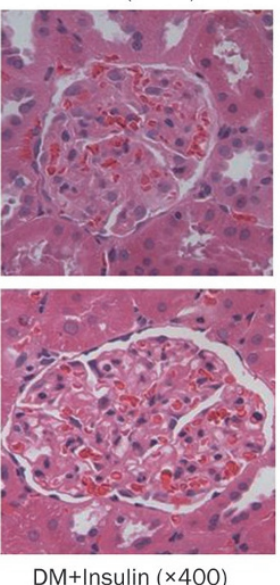

$\mathrm{DM}(\times 400)$

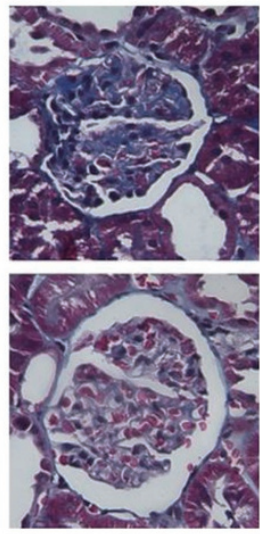

DM+Insulin (×400)
B

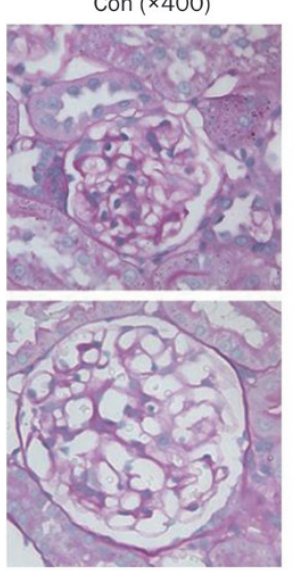

$\mathrm{DM}+\mathrm{PPP}(\times 400)$
$\mathrm{DM}(\times 400)$
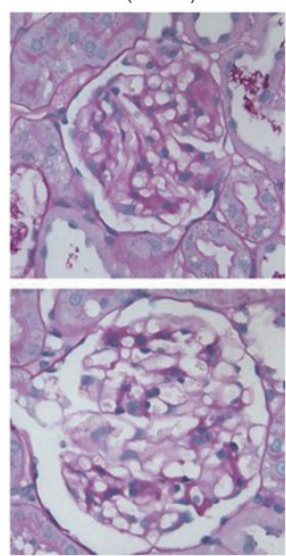

$\mathrm{DM}+$ Insulin $(\times 400)$

D Con $(\times 1500)$ Con $(\times 4000)$

DM $(\times 1500) \quad$ DM $(\times 4000)$
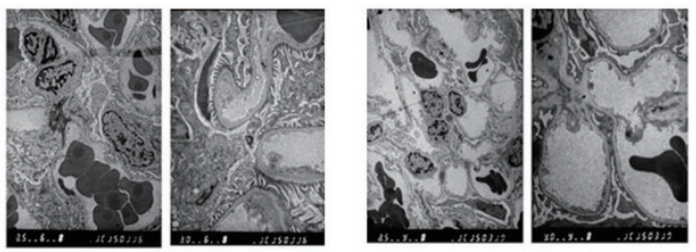

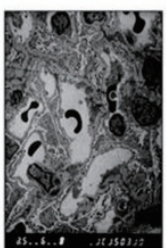

$\mathrm{DM}+\mathrm{PPP}$

$(\times 1500)$

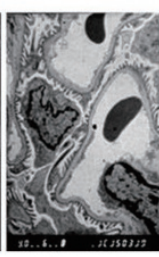

$\mathrm{DM}+\mathrm{PPP}$

$(\times 4000)$

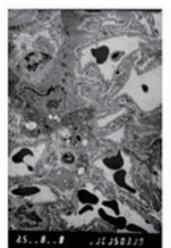

$\mathrm{DM}+$ Insulin $(\times 1500)$

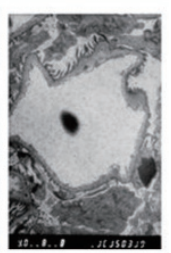

$\mathrm{DM}+$ Insulin $(\times 4000)$

Figure 9. Effects of IGF-1R blockade on renal morphology in normal and diabetic rats. At the end of the study (12 weeks after STZ injection), kidney histology was examined by HE (A), PAS (B) and Masson's trichrome staining (C). Original magnifications $\times 400$. (D) Part of the right kidney was fixed in $2.5 \%$ glutaraldehyde for electron microscopy, and the ultrastructure (glomerulus basement membrane and podocyte processes) of the kidney was observed at magnifications of $\times 1500$ and $\times 4000$, respectively. 
that there is IGF-1R expression in the kidney ${ }^{[2]}$. Therefore, we speculate that the IGF-1 and IGF-1R pathways might be involved in regulating the function of MCs. The present study showed that ID increased the expression of IR, IGF-1 and IGF$1 R$, and the phosphorylation of IGF-1R was also increased. Pretreatment with PPP, a blocker of IGF-1R, antagonized the ID-induced increase in IGF-1R phosphorylation and reversed the ID-induced MC proliferation and fibrosis-related gene expression. These results suggested that the activation of the IGF-1 and IGF-1R pathways is related to ID-induced abnormalities in MC proliferation and increase in ECM synthesis. Knockdown of IGF-1R expression by siRNA showed a similar effect to the application of PPP. Furthermore, in the current study, exogenous IGF-1 significantly promoted MC proliferation. These results confirmed that the activation of IGF-1R stimulates proliferation and ECM synthesis in renal MCs.

To further verify the role of IGF-1 and IGF-1R pathway in ID-induced renal injury, an STZ-induced diabetic rat, which is a classic animal model of ID, was utilized in this study. STZ specifically destroys the $\beta$ cells in the islets of the pancreas, which results in an absolute lack of insulin and leads to the occurrence of diabetes ${ }^{[34]}$. In the present study, blood glucose, urine volume, urinary glucose and kidney weight in the STZinjected rats were significantly higher than in the control group. Similar to the observations from the in vitro study, the expression of IR, IGF-1 and IGF-1R was significantly elevated in the diabetic rats. The administration of the IGF-1R blocker PPP significantly reduced kidney weight, which is a sign of early stage DN, while no obvious changes were observed for body weight, blood glucose levels, urine volume and urinary glucose. Moreover, the increased urinary protein output, BUN and plasma creatinine, as well as the ECM expression and TGF- $\beta$ synthesis, were significantly attenuated. These results suggested that blockade of IGF-1R does not affect blood glucose in diabetic rats, but slows down the development of DN.

Supplementation of exogenous insulin in the diabetic rat also showed a similar protective effect. However, treatment of the animal with either insulin or IGF-1R blocker did not completely retard the occurrence of DN. The reason for the IGF$1 \mathrm{R}$ blockade results might be that it did not eliminate the effect of high blood glucose. The reason for the insulin supplementation results, we would speculate, is due to a failure to stably control blood glucose. In the clinic, a major obstacle in the treatment of diabetes is that exogenous supplementation cannot restore the blood glucose level to the endogenous secretion level ${ }^{[35]}$. In this experiment, we noticed that a decrease in the blood glucose was observed $4 \mathrm{~h}$ after the insulin injection but not at $12 \mathrm{~h}$. Urinary glucose was detected, and the urine volume and kidney weight were only partially decreased in the DM+insulin group.

In this experiment, the renal morphological observations showed that there was little MC proliferation, a slight mesangial matrix increase, glomerular basement membrane thickening, mesangial matrix accumulation, plasma protein extravasation and partial epithelial foot processes fusion in the DM group, and all of these features are principle pathological changes in DN. The morphological changes were improved slightly in the DM+PPP and the DM+insulin groups. Overall, the subjected animals were still in the early stages of DN, and the morphological differences among the groups were not obvious. To confirm the morphological effects, studies exploring prolonged treatment are required.

In summary, the renal IGF-1/ IGF-1R pathway is upregulated and activated under an insulin-deficient condition. The activation of the IGF-1/IGF-1R pathway contributes to the development of DN. The stimulation of renal MC proliferation and ECM production is involved in the IGF-1/IGF-1R pathway activation-induced renal damage. Blockade of the IGF-1/IGF-1R pathway activation might be a potential target in manipulating DN, and an early application of insulin might be recommended in the clinic to prevent the overactivation of the IGF-1/IGF-1R pathway.

\section{Acknowledgements}

This research was financially supported by the National Natural Science Foundation of China (№ 81470591 and 81170636 to Li-min LU, and № 81100531 to Wei ZHANG).

\section{Author contribution}

Wei ZHANG, Xiao-xia WANG and Li-min LU designed the research; Ya-li KONG and Yang SHEN performed the experiments; Jun NI and De-cui SHAO analyzed the data; Nai-jun MIAO and Jin-lan XU contributed the reagents and materials; Ya-li KONG, Li ZHOU, and Hong XUE wrote the manuscript. Ya-li KONG, Xiao-xia WANG and Li-min LU revised the manuscript.

\section{References}

1 Kolset SO, Reinholt FP, Jenssen T. Diabetic nephropathy and extracellular matrix. J Histochem Cytochem 2012; 60: 976-86.

2 Kanwar YS, Wada J, Sun L, Xie P, Wallner El, Chen S, et al. Diabetic nephropathy: mechanisms of renal disease progression. Exp Biol Med (Maywood) 2008; 233: 4-11.

3 Brunskill EW, Potter SS. Changes in the gene expression programs of renal mesangial cells during diabetic nephropathy. BMC Nephrol 2012; 13: 70.

4 Shao D, Liu J, Ni J, Wang Z, Shen Y, Zhou L, et al. Suppression of XBP1S mediates high glucose-induced oxidative stress and extracellular matrix synthesis in renal mesangial cell and kidney of diabetic rats. PloS One 2013; 8: e56124.

5 Li JJ, Lee SH, Kim DK, Jin R, Jung DS, Kwak SJ, et al. Colchicine attenuates inflammatory cell infiltration and extracellular matrix accumulation in diabetic nephropathy. Am J Physiol Renal Physiol 2009; 297: F200-9.

6 Lupia E, Elliot SJ, Lenz O, Zheng F, Hattori M, Striker GE, et al. IGF1 decreases collagen degradation in diabetic NOD mesangial cells: implications for diabetic nephropathy. Diabetes 1999; 48: 1638-44.

7 Wang Z, Ni J, Shao D, Liu J, Shen Y, Zhou L, et al. Elevated transcriptional co-activator p102 mediates angiotensin II type 1 receptor up-regulation and extracellular matrix overproduction in the high glucose-treated rat glomerular mesangial cells and isolated glomeruli. Eur J Pharmacol 2013; 702: 208-17.

8 Yuan P, Xue H, Zhou L, Qu L, Li C, Wang Z, et al. Rescue of mesangial cells from high glucose-induced over-proliferation and extracellular 
matrix secretion by hydrogen sulfide. Nephrol Dial Transplant 2011; 26: $2119-26$

9 Xue H, Yuan P, Ni J, Li C, Shao D, Liu J, et al. $\mathrm{H}_{2} \mathrm{~S}$ inhibits hyperglycemia-induced intrarenal renin-angiotensin system activation via attenuation of reactive oxygen species generation. PLoS One 2013; 8: e74366.

10 Shao D, Liu J, Ni J, Wang Z, Shen Y, Zhou L, et al. Suppression of XBP1S mediates high glucose-induced oxidative stress and extracellular matrix synthesis in renal mesangial cell and kidney of diabetic rats. PLoS One 2013; 8: e56124.

11 Xue H, Zhou L, Yuan P, Wang Z, Ni J, Yao T, et al. Counteraction between angiotensin II and angiotensin-(1-7) via activating angiotensin type I and Mas receptor on rat renal mesangial cells. Regul Pept 2012; 177: 12-20.

12 Xue H, Yuan P, Zhou L, Yao T, Huang Y, Lu LM. Effect of adrenotensin on cell proliferation is mediated by angiotensin II in cultured rat mesangial cells. Acta Pharmacol Sin 2009; 30: 1132-7.

13 Gwaltney SN. Current approaches to the treatment of diabetes. Curr Top Med Chem 2008; 8: 1482.

14 Zeng R, Xiong Y, Zhu F, Ma Z, Liao W, He Y, et al. Fenofibrate attenuated glucose-induced mesangial cells proliferation and extracellular matrix synthesis via PI3K/AKT and ERK1/2. PLoS One 2013; 8: e76836.

15 Peti-Peterdi J. High glucose and renin release: the role of succinate and GPR91. Kidney Int 2010; 78: 1214-7.

16 Arora MK, Singh UK. Oxidative stress: meeting multiple targets in pathogenesis of diabetic nephropathy. Curr Drug Targets 2014; 15 : 531-8.

17 Balakumar P, Arora MK, Reddy J, Anand-Srivastava MB. Pathophysiology of diabetic nephropathy: involvement of multifaceted signalling mechanism. J Cardiovasc Pharmacol 2009; 54: 129-38.

18 Fukami K, Yamagishi S, Coughlan MT, Harcourt BE, Kantharidis P, Thallas-Bonke $\mathrm{V}$, et al. Ramipril inhibits AGE-RAGE-induced matrix metalloproteinase-2 activation in experimental diabetic nephropathy. Diabetol Metab Syndr 2014; 6: 86.

$19 \mathrm{Hu}$ J, Klein JD, Du J, Wang XH. Cardiac muscle protein catabolism in diabetes mellitus: activation of the ubiquitin-proteasome system by insulin deficiency. Endocrinology 2008; 149: 5384-90.

20 Wang Y, Deb DK, Zhang Z, Sun T, Liu W, Yoon D, et al. Vitamin D receptor signaling in podocytes protects against diabetic nephropathy. J Am Soc Nephrol 2012; 23: 1977-86.

21 Haider S, Ahmed S, Tabassum S, Memon Z, Ikram M, Haleem DJ. Streptozotocin-induced insulin deficiency leads to development of behavioral deficits in rats. Acta Neurol Belg 2013; 113: 35-41.

22 Ye R, Holland WL, Gordillo R, Wang M, Wang QA, Shao M, et al.
Adiponectin is essential for lipid homeostasis and survival under insulin deficiency and promotes beta-cell regeneration. Elife 2014; 3. doi: 10.7554/eLife.03851.

23 Ley EJ, Srour MK, Clond MA, Barnajian M, Tillou A, Mirocha J, et al. Diabetic patients with traumatic brain injury: insulin deficiency is associated with increased mortality. J Trauma 2011; 70: 1141-4.

24 Bayat AH, Haghparast A. Effect of insulin deficiency on the rewarding properties of methamphetamine in streptozotocin-induced diabetic rats. Pharmacol Biochem Behav 2015; 128: 8-13.

25 Ullrich A, Bell JR, Chen EY, Herrera R, Petruzzelli LM, Dull TJ, et al. Human insulin receptor and its relationship to the tyrosine kinase family of oncogenes. Nature 1985; 313: 756-61.

26 Singh P, Alex JM, Bast F. Insulin receptor (IR) and insulin-like growth factor receptor 1 (IGF-1R) signaling systems: novel treatment strategies for cancer. Med Oncol 2014; 31: 805.

27 Weroha SJ, Haluska P. The insulin-like growth factor system in cancer. Endocrinol Metab Clin North Am 2012; 41: 335-50.

28 Kawai M, Rosen CJ. The insulin-like growth factor system in bone: basic and clinical implications. Endocrinol Metab Clin North Am 2012; 41: 323-33.

29 Rabkin R, Schaefer F. New concepts: growth hormone, insulin-like growth factor-I and the kidney. Growth Horm IGF Res 2004; 14: $270-$ 6.

30 Chu CH, Tzang BS, Chen LM, Kuo CH, Cheng YC, Chen LY, et al. IGFII/mannose-6-phosphate receptor signaling induced cell hypertrophy and atrial natriuretic peptide/BNP expression via Galphaq interaction and protein kinase $\mathrm{C}$-alpha/CaMKII activation in $\mathrm{H} 9 \mathrm{c} 2$ cardiomyoblast cells. J Endocrinol 2008; 197: 381-90.

31 Haisa M. The type 1 insulin-like growth factor receptor signalling system and targeted tyrosine kinase inhibition in cancer. J Int Med Res 2013; 41: 253-64.

32 Estrada JA, Contreras I, Pliego-Rivero FB, Otero GA. Molecular mechanisms of cognitive impairment in iron deficiency: alterations in brain-derived neurotrophic factor and insulin-like growth factor expression and function in the central nervous system. Nutr Neurosci 2014; 17: 193-206.

33 Dupraz S, Grassi D, Karnas D, Nieto GA, Hicks D, Quiroga S. The insulin-like growth factor 1 receptor is essential for axonal regeneration in adult central nervous system neurons. PLoS One 2013; 8: e54462.

34 Szkudelski T. The mechanism of alloxan and streptozotocin action in B cells of the rat pancreas. Physiol Res 2001; 50: 537-46.

35 ter Wee PM, Donker JM. Diabetic nephropathy in insulin-dependent diabetic patients: renal hemodynamics and derived treatment strategies. J Diabet Complications 1989; 3: 62-9. 